\title{
artigo
}

Paixão, L.S.S.; Mendonça, R.P.

Desafios dos enfermeiros frente á aplicabilidade da sistematização da assistência de enfermagem: revisão integrativa

\section{Desafios dos enfermeiros frente á aplicabilidade da sistematização da assistência de enfermagem: revisão integrativa}

Challenges of nurses in front of the applicability of nursing care systematization: integrative review

Retos de las enfermeras frente a la aplicabilidad de la sistematización de la atención de enfermería: revisión integradora

\section{RESUMO}

Objetivo: analisar quais os desafios que limitam os Enfermeiros na aplicação da Sistematização da Assistência de Enfermagem. Método: Revisão integrativa realizada na BVS por artigos publicados em periódicos nacionais, utilizando-se as bases de dados LILACS, BDENF e MEDLINE, com os descritores Processo de enfermagem; Enfermeiros; Hospital; Conhecimento e Assistência de enfermagem. Foram analisados artigos em português e com recorte temporal de 2010 a 2020. Resultados: "sobrecarga de trabalho", "falta de conhecimento", "necessidade de aprimoramento" e "falta de apoio da chefia/instituição" foram mencionados com mais frequência possuindo um reflexo negativo na aplicabilidade da SAE. Destes emergiram dois categoriais argumentados na discussão. Conclusão: a aplicabilidade desfalcada e superficial da SAE, exercida em algumas unidades de saúde, são caracterizadas pelas dificuldades identificadas, desde a formação dos Enfermeiros no curso de graduação, até condições de trabalho que prejudicam o êxito da execução do método.

DESCRITORES: Processo de Enfermagem; Enfermeiros; Hospital; Conhecimento; Assistência de Enfermagem.

\section{ABSTRACT}

Objective: to analyze the challenges that limit Nurses in the application of the Systematization of Nursing Assistance. Method: Integrative review carried out in the VHL by articles published in national journals, using the databases LILACS, BDENF and MEDLINE, with the keywords Nursing process; Nurses; Hospital; Nursing Knowledge and Assistance. Articles in Portuguese were analyzed and with a time frame from 2010 to 2020. Results: "work overload", "lack of knowledge", "need for improvement" and "lack of support from the management / institution" were mentioned more frequently, having a negative impact on the applicability of SAE. From these two categories emerged argued in the discussion. Conclusion: the lacking and superficial applicability of $S A E$, exercised in some health units, are characterized by the difficulties identified, from the training of nurses in the undergraduate course, to working conditions that hinder the successful execution of the method.

DESCRIPTORS: Nursing Process; Nurses; Hospital; Knowledge; Nursing Assistance.

\section{RESUMEN}

Objetivo: analizar los desafíos que limitan al Enfermero en la aplicación de la Sistematización de la Atención de Enfermería. Método: Revisión integrativa realizada en la BVS por artículos publicados en revistas nacionales, utilizando las bases de datos LILACS, BDENF y MEDLINE, con las palabras clave Proceso de enfermería; Enfermeras; Hospital; Conocimientos y asistencia en enfermería. Se analizaron artículos en portugués y con un marco temporal de 2010 a 2020. Resultados: se mencionaron con mayor frecuencia "sobrecarga de trabajo", "falta de conocimiento", "necesidad de mejora" y "falta de apoyo de la dirección / institución", habiendo un impacto negativo en la aplicabilidad de SAE. De estas dos categorías surgieron argumentadas en la discusión. Conclusión: la escasa y superficial aplicabilidad de los SAE, ejercidos en algunas unidades de salud, se caracterizan por las dificultades identificadas, desde la formación de enfermeros en la carrera de pregrado, hasta condiciones laborales que dificultan la ejecución exitosa del método. DESCRIPTORES: Proceso de Enfermería; Enfermeras; Hospital; Conocimiento; Asistencia de enfermería.

RECEBIDO EM: 27/01/2021 APROVADO EM: 15/03/2021

\section{Luiza dos Santos Souza Paixão}

Acadêmica de Enfermagem do sétimo período pela Universidade Salgado de Oliveira.

ORCID: 0000-0002-9477-436X 


\section{Rita Patrizzi Mendonça}

Enfermeira. Especialista em Terapia intensiva com ênfase em Cardiologia pela Universidade Federal Fluminense. Mestre em Gerência do Cuidado pelo Programa de Mestrado Profissional da Universidade Federal Fluminense. Professora titular da Universidade Salgado de Oliveira. ORCID: 0000-0002-8466-989X

\section{INTRODUÇ̃̃O}

A Sistematização da Assistência de Enfermagem (SAE) é regulamentada no Brasil como um método que organiza o trabalho profissional, possibilitando a implementação do Processo de Enfermagem (PE), instrumento metodológico que orienta o cuidado profissional de enfermagem, organizado em cinco etapas inter-relacionadas: coleta de dados, diagnóstico de enfermagem (NANDA), planejamento (NOC), implementação (NIC) e avaliação de enfermagem ${ }^{(1)}$. O Processo de Enfermagem aparece como instrumento de trabalho do enfermeiro, na organização, no planejamento, na execução e avaliação desta ação ${ }^{(2)}$.

Segundo a Resolução do COFEN $358 / 2009^{(3)}$ : as etapas da SAE devem ser realizadas, de modo deliberado e sistemático, em todos os ambientes, públicos ou privados, em que ocorre o cuidado profissional de Enfermagem através de suas cinco etapas operacionais. Desta forma a SAE permite ao enfermeiro planejar e tomar decisões conjuntas com a equipe de enfermagem identificando as necessidades de diversas ordens, por parte dos serviços, da própria equipe e dos usuários, contribuindo para a melhoria efetiva da resolução das problemáticas da prática diária.

Os enfermeiros usam a avaliação e o julgamento clínico para formular hipóteses ou explicações sobre os problemas, riscos e/ou oportunidades de promoção da saúde que se apresentam. Todas essas etapas exigem conhecimento de conceitos subjacentes à ciência da enfermagem antes da identificação de padrões nos dados clínicos ou da elaboração de diagnósticos exatos ${ }^{(4)}$. Sendo assim, a aplicação da SAE é a única possibilidade de o enfermeiro atingir sua autonomia profissional e constitui a essência de sua práxis, considera-se que a sistematização da assistência é de fundamental importância para o fortalecimento e reconhecimento das equipes de enfermagem ${ }^{(5,6)}$.

É notório que o método contribui para a melhoria do trabalho, organiza os registros das ações de enfermagem, valoriza e direciona o trabalho da equipe e promove mais interação entre a equipe, o paciente e familiares. No entanto, a adesão ao seu uso não está consolidada. Foram aludidos alguns desafios para essa adesão, bem como algumas inadequações do instrumento da SAE ${ }^{(7)}$. Percebe-se que os profissionais não colocam em prática todas as etapas da SAE em seu ambiente de trabalho, pois esta implica aprimoramento na utilização dessa metodologia para a sua implementação, o que requer constante atualização, habilidades e experiência ${ }^{(8)}$.

É necessário avaliar todos os aspectos que possam contribuir para a sua implantação, e conhecer os pontos que a prejudicam, pois, apesar das diversas vantagens proporcionadas pela mesma, existem problemas encontrados na sua execução, como, número insuficiente de profissionais; falta de capacitação; impressos inadequados; recursos escassos; tempo minimizado, sobretudo, na unidade hospitalar onde ele significa vida ou morte; além da ausência de padronização da linguagem, fator primordial para o seu funcionamento ${ }^{(9)}$.

Diante do exposto, o objetivo deste estudo é analisar quais os desafios que limitam os Enfermeiros na aplicação da Sistematização da Assistência de Enfermagem. Espera-se que os resultados deste estudo sejam satisfatórios no sentido de propor uma reflexão e pensamento crítico acerca da temática, bem como o incentivo de novos métodos e estudos que facilitem a devida implementação e possibilite o êxito no desenvolvimento profissional da Enfermagem.

\section{MÉTODO}

Trata-se de uma revisão integrativa que consiste em um método de pesquisa que analisa amplamente os estudos, com o objetivo de sintetizar as ideias expostas, contribuindo para a discussão e resultados da pesquisa, bem como para preencher as lacunas encontradas com a formulação de novos trabalhos ${ }^{(10)}$. Para construção desta revisão, foram seguidas as seguintes etapas: identificar o objetivo da pesquisa; critérios de inclusão e exclusão; busca na literatura; análise crítica dos estudos incluídos; discussão do tema e apresentação da revisão integrativa. $\mathrm{O}$ levantamento bibliográfico foi realizado no período de novembro á dezembro de 2020 na Biblioteca Virtual de Saúde objetivando artigos publicados em periódicos nacionais e utilizando as três bases de dados: BDENF (Base de dados de Enfermagem), LILACS (Literatura Latino-americana e do Caribe em Ciências da Saúde) e MEDLINE, no intuito de ampliar o campo de pesquisa. Os critérios de inclusão foram: artigos em português, texto completo disponível gratuitamente nas bases de dados que foram selecionadas, com recorte temporal de 2010 a 2020, cuja metodologia contemplava o objetivo da pesquisa, excluindo estudos duplicados e que não apresentava conteúdo a fim de contemplar o objetivo central. Foram utilizados os descritores "Processos de enfermagem"; "Enfermeiros"; "Hospital"; "Conhecimento" e "Assistência de enfermagem", localizados no DECS. Para realização de cruzamentos dos descritores, fez-se uso do operador boleano "AND" objetivando artigos específicos acerca do tema. Foram elegidos um total de 12 artigos que atenderam aos critérios do estudo. Destes resultados emergiram dois categoriais: "Consequências da aplicabilidade errônea" e "Resta- 


\section{artigo}

Paixão, L.S.S.; Mendonça, R.P.

Desafios dos enfermeiros frente á aplicabilidade da sistematização da assistência de enfermagem: revisão integrativa

belecimento da ordem através da educação continuada", que serão argumentadas na discussão.

\section{RESULTADOS}

Os resultados foram apresentados em duas etapas, sendo a primeira através da descrição dos estudos selecionados (quadro 1) onde consta: caracterização dos artigos selecionados conforme título, ano de publicação, local de estudo, delineamento e nível de evidência científica. A segunda etapa foi apresentada de forma descritiva, as quais são relatados os desafios e dificuldades enfrentadas pelos profissionais de
Figura 1: Seleção de artigos e cruzamento dos descritores utilizando operador boleano.
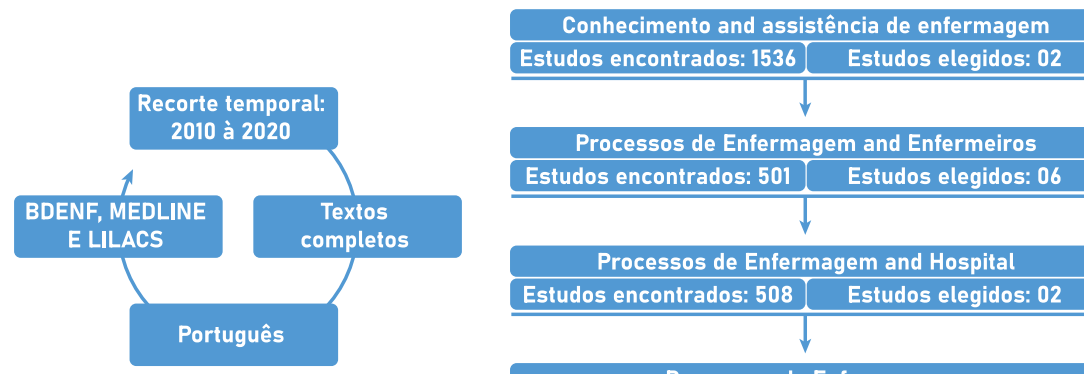

Processos de Enfermagem and Enfermeiros Estudos encontrados: 501 Estudos elegidos: 06

Processos de Enfermagem and Hospital Estudos encontrados: 508 Estudos elegidos: 02

Processos de Enfermagem Estudos encontrados: $1344 \quad$ Estudos elegidos: 02

Fonte: autoria própria (2020).

enfermagem citados com maior frequência nos periódicos e que inibem a implementação adequada da SAE.

Dos 12 artigos analisados, foram identificadas de acordo com os resultados as causas mais citadas que podem ser consideradas objeções limitando os profissionais Enfermeiros á não implementação da SAE de maneira eficaz. São estas: "sobrecarga de trabalho" constando em 33,32\% (n.4) dos artigos analisados; "falta de conhecimento" em 33,32\% (n.4) artigos; "necessidade de aprimoramento" citado em 33,32\% (n.4) artigos e "falta de apoio da chefia/instituição" relatado em 24,99\% (n.3) artigos.

\section{DISCUSSÃO}

A partir da análise do conjunto dos principais resultados obtidos dos 12 artigos selecionados nesta revisão, emergiram duas categorias: "Consequências da aplicabilidade errônea" e "Restabelecimento da ordem através da educação continuada”.

QUADRO 01- Caracterização dos artigos selecionados conforme título, ano de publicação, local de estudo, delineamento e nível de evidência científica (segundo a Classificação de Oxford Centre for Evidence-Based Medicine). Rio de janeiro, Brasil, 2020.

\begin{tabular}{|c|c|c|c|c|c|}
\hline $\mathbf{N}^{0}$ & TÍTULO DO ARTIGO & ANO & LOCAL DO ESTUDO & DELINEAMENTO & $\begin{array}{l}\text { NIIVEL DE } \\
\text { EVIDÊNCIA }\end{array}$ \\
\hline 1 & $\begin{array}{l}\text { Conhecimento dos pro- } \\
\text { fissionais de enfermagem } \\
\text { sobre a sistematização da } \\
\text { assistência de enferma- } \\
\text { gem. }\end{array}$ & 2019 & $\begin{array}{l}\text { Hospital universitário de } \\
\text { grande porte localizado no } \\
\text { município de Petrolina/PE. } \\
\text { Brasil. }\end{array}$ & $\begin{array}{l}\text { Estudo descritivo, analítico, exploratório com } \\
\text { abordagem quantitativa, constituída por } 105 \\
\text { profissionais de enfermagem através de } \\
\text { questionário semiestruturado cujos dados } \\
\text { foram apresentados por estatística descritiva } \\
\text { e analítica adotando-se } 5 \% \text { de significância } \\
\text { e } 95 \% \text { de confiança e utilizados os testes de } \\
\text { Mann-Whitney, Exato de Fisher e Qui-qua- } \\
\text { drado de Person para associação bivariada. }\end{array}$ & 4 \\
\hline 2 & $\begin{array}{c}\text { Ações coordenadas } \\
\text { para implantação } \\
\text { e consolidação da } \\
\text { sistematização } \\
\text { da assistência de } \\
\text { Enfermagem. }\end{array}$ & 2019 & $\begin{array}{l}\text { Universidade Federal } \\
\text { Ciências da Saúde de Porto } \\
\text { Alegre-UFCSPA. RS. Brasil. }\end{array}$ & $\begin{array}{c}\text { Relatório técnico realizado por docentes e } \\
\text { discente alinhado ao ensino de graduação e } \\
\text { residência multiprofissional e de sua relação } \\
\text { com serviço de saúde ao qual fomentou } \\
\text { propostas de projetos de intervenção e } \\
\text { pesquisa, gerando produtos da SAE pelos } \\
\text { mestrandos. }\end{array}$ & 4 \\
\hline 3 & $\begin{array}{l}\text { Percepções das equipes de } \\
\text { enfermagem na atenção } \\
\text { básica frente à sistema- } \\
\text { tização da assistência de } \\
\text { Enfermagem. }\end{array}$ & 2019 & $\begin{array}{l}\text { Unidade de Atenção Básica. } \\
\text { Criciúma, Santa Catarina, } \\
\text { Brasil. }\end{array}$ & $\begin{array}{l}\text { Estudo transversal, com abordagem quanti- } \\
\text { tativa, realizado com } 76 \text { profissionais de en- } \\
\text { fermagem, por meio de questionário com } 66 \\
\text { questões objetivas, vinculadas aos aspectos } \\
\text { sócios demográficos e aos } 7 \text { domínios sobre a } \\
\text { percepções da assistência de enfermagem e } \\
\text { do processo de enfermagem. }\end{array}$ & 4 \\
\hline
\end{tabular}




\begin{tabular}{|c|c|c|c|c|c|}
\hline 4 & $\begin{array}{l}\text { Percepção de enfermeiros } \\
\text { de unidades de internação } \\
\text { clínica sobre a Sistema- } \\
\text { tização da Assistência de } \\
\text { Enfermagem. }\end{array}$ & 2019 & $\begin{array}{l}\text { Hospital universitário de } \\
\text { Porto Alegre, RS. Brasil. }\end{array}$ & $\begin{array}{l}\text { Estudo descritivo, com abordagem qualita- } \\
\text { tiva, realizado com } 18 \text { enfermeiros por meio } \\
\text { de entrevista semiestruturada ao qual foram } \\
\text { coletados dados acerca do conhecimento, com- } \\
\text { preensão, utilização, dificuldades, vantagens e } \\
\text { desvantagens da SAE. }\end{array}$ & 4 \\
\hline 5 & $\begin{array}{l}\text { Sistematização da assis- } \\
\text { tência de enfermagem: } \\
\text { ordem, desordem ou (re) } \\
\text { organização? }\end{array}$ & 2017 & $\begin{array}{l}\text { Duas Instituições hospitala- } \\
\text { res de médio porte na região } \\
\text { central do Rio Grande do Sul. } \\
\text { Brasil. }\end{array}$ & $\begin{array}{c}\text { Estudo qualitativo, descritivo, exploratório } \\
\text { com } 20 \text { enfermeiros assistenciais por meio de } \\
\text { entrevistas individuais, com cinco questões } \\
\text { norteadoras e duas complementares acerca } \\
\text { da: Percepção sobre a SAE; Implementação da } \\
\text { SAE na unidade; Estratégia de implementação; } \\
\text { Utilização de alguma teoria para fundamentar o } \\
\text { processo. }\end{array}$ & 4 \\
\hline 6 & $\begin{array}{l}\text { Percepção da equipe de } \\
\text { enfermagem quanto à } \\
\text { sistematização da assis- } \\
\text { tência de enfermagem em } \\
\text { um serviço de emergência } \\
\text { psiquiátrica. }\end{array}$ & 2016 & $\begin{array}{l}\text { Serviço de emergência } \\
\text { psiquiátrica de uma cidade do } \\
\text { interior de São Paulo. Brasil. }\end{array}$ & $\begin{array}{l}\text { Estudo qualitativo transversal de caráter } \\
\text { descritivo e exploratório com } 15 \text { profissionais } \\
\text { de Enfermagem onde utilizou entrevistas } \\
\text { objetivando identificar a percepção a respeito da } \\
\text { "Sistematização da Assistência de Enfermagem" } \\
\text { na unidade. }\end{array}$ & 4 \\
\hline 7 & $\begin{array}{l}\text { Sistematização da assis- } \\
\text { tência de Enfermagem } \\
\text { na perspectiva da equipe } \\
\text { de enfermagem de um } \\
\text { hospital público do norte } \\
\text { do Brasil. }\end{array}$ & 2019 & $\begin{array}{l}\text { Maternidade na região cen- } \\
\text { tral de Belém, Pará. Brasil. }\end{array}$ & $\begin{array}{l}\text { Pesquisa descritiva, qualitativa, realizada com } \\
40 \text { enfermeiros e técnicos de enfermagem } \\
\text { com a utilização de entrevista semiestruturada } \\
\text { acerca da SAE cujo resultado foi interpretado } \\
\text { sob análise do conteúdo de Bardin. }\end{array}$ & 4 \\
\hline 8 & $\begin{array}{c}\text { Autonomia e vulnerabi- } \\
\text { lidade do enfermeiro na } \\
\text { prática da Sistematização } \\
\text { da Assistência de Enfer- } \\
\text { magem. }\end{array}$ & 2011 & São Paulo, SP. Brasil. & $\begin{array}{c}\text { Pesquisa bibliográfica sistemática e qualitativa } \\
\text { a partir da análise do conteúdo de artigos de } 40 \\
\text { periódicos científicos objetivando reconhecer a } \\
\text { autonomia e a vulnerabilidade do enfermeiro no } \\
\text { processo de implantação e implementação da } \\
\text { SAE. }\end{array}$ & 4 \\
\hline 9 & $\begin{array}{l}\text { Conhecimento das enfer- } \\
\text { meiras de ambulatórios de } \\
\text { hemofilia sobre a sistema- } \\
\text { tização da assistência de } \\
\text { enfermagem. }\end{array}$ & 2016 & $\begin{array}{l}\text { Ambulatórios de hemofilia } \\
\text { nos municípios de Fortale- } \\
\text { za, Crato, Sobral, Quixadá e } \\
\text { Iguatu no Estado do Ceará. } \\
\text { Brasil. }\end{array}$ & $\begin{array}{c}\text { Estudo descritivo, com abordagem qualitativa } \\
\text { com } 9 \text { Enfermeiros através de questionários } \\
\text { com o objetivo de avaliar o conhecimento das } \\
\text { enfermeiras sobre a SAE. }\end{array}$ & 5 \\
\hline 10 & $\begin{array}{l}\text { Sistematização da Assis- } \\
\text { tência de Enfermagem em } \\
\text { instituições de saúde no } \\
\text { Brasil: revisão integrativa. }\end{array}$ & 2015 & $\begin{array}{l}\text { Rio Grande do Norte, Natal. } \\
\text { Brasil. }\end{array}$ & $\begin{array}{l}\text { Revisão integrativa com } 11 \text { artigos sendo estes } \\
\text { classificados de acordo com seu nível de evidên- } \\
\text { cia, cujo objetivo era de analisar a implementação } \\
\text { da Sistematização da Assistência em Enferma- } \\
\text { gem em Instituições de Saúde Brasileiras. }\end{array}$ & 4 \\
\hline 11 & $\begin{array}{l}\text { Avaliação da implantação } \\
\text { da sistematização da } \\
\text { assistência de enferma- } \\
\text { gem em uma unidade } \\
\text { pediátrica. }\end{array}$ & 2012 & $\begin{array}{l}\text { Hospital público universitário } \\
\text { de grande porte, localizado } \\
\text { no estado de Minas Gerais, } \\
\text { MG. Brasil. }\end{array}$ & $\begin{array}{c}\text { Estudo de caso descritivo e exploratório, de } \\
\text { abordagem quantitativa, sobre o processo de } \\
\text { implantação da SAE com um total de } 79 \text { pro- } \\
\text { fissionais de Enfermagem sob forma de coleta } \\
\text { de dados através de formulários institucionais } \\
\text { e questionários cujo objetivo foi o foi avaliar o } \\
\text { processo de implantação da SAE. }\end{array}$ & 5 \\
\hline 12 & $\begin{array}{c}\text { Sistematização da assis- } \\
\text { tência de enfermagem: } \\
\text { dificuldades evidenciadas } \\
\text { pela teoria fundamentada } \\
\text { nos dados. }\end{array}$ & 2013 & $\begin{array}{l}\text { Maternidade pública do } \\
\text { município de João Pessoa, } \\
\text { Paraíba. }\end{array}$ & $\begin{array}{l}\text { Pesquisa qualitativa na perspectiva da Teoria } \\
\text { Fundamentada nos Dados através de entrevista } \\
\text { aberta, com } 13 \text { enfermeiros cujo objetivo foi de } \\
\text { investigar os fatores que dificultam a operacio- } \\
\text { nalização da SAE no serviço de obstetrícia. }\end{array}$ & 4 \\
\hline
\end{tabular}




\section{Consequências da aplicabilidade er- rônea}

Apesar da Sistematização da Assistência ser imposta uma vez que faz parte do exercício profissional do Enfermeiro, existem muitos fatores identificados nesta revisão que caracteriza a dificuldade de execução da mesma. A sobrecarga de trabalho (N. 4; 33,32\%) e falta de apoio da chefia/ instituição (N. 3; 24,99\%) enfatizam a necessidade de uma reformulação no âmbito profissional de algumas unidades de saúde reforçando-se através da própria aplicabilidade da SAE que como consequência desta adesão ao método, resultará em atendimentos mais eficazes através de uma assistência individualizada.

Percebe se que, a sobrecarga de trabalho do enfermeiro, associada ao número reduzido de profissionais de enfermagem nas instituições de saúde, tem interferido diretamente na aplicação do processo de enfermagem. Os enfermeiros exercem muitas atribuições nem sempre ligadas à sua área de atuação profissional, fato que os distancia da assistência, acarretando uma carga excessiva de trabalho. Em consequência, a execução do processo de enfermagem deixa de ser prioridade ${ }^{(11)}$.

$O$ processo de implantação da $\mathrm{SAE}$ deve ocorrer num contexto de gestão participativa e considerar aspectos organizacionais, como número de funcionários e intensidade de cuidado demandado pelos pacientes da unidade ${ }^{(12)}$. Com isso, as consequências da não adesão a SAE e PE podem refletir em desfechos desfavoráveis à saúde do indivíduo: maiores chances de complicações, eventos adversos, maior tempo de internação hospitalar, maiores custos, maiores transtornos na rotina do paciente e família e alterações psicoemocionais decorrentes do processo de hospitalização ${ }^{(13)}$.

A SAE ainda se reduz, em parte, às questôes de ordem, a normas e rotinas, e que, por vezes, instaura certa desordem, a qual possibilita gradativamente nova organização. Com isso, há que se pensar em estratégias para modificar esse pensamento simplificador e, excessivamente, regulador do processo de trabalho do enfermeiro ${ }^{(14)}$.
A ausência de estímulo e apoio das instâncias superiores representa vulnerabilidade, pois está sujeita ao desconhecimento associado ao não envolvimento, não compromisso, não valorização de sua execução na prática e também a não capacitação para execução da SAE, bem como lacunas na formação pelo ensino formal e dificuldades estruturais institucionais. Contudo, o interesse em realizar as ações com compromisso ético, moral e com responsabilidade, independente dos desafios que o cotidiano impõe na atuação profissional, contribui para uma prática autônoma. ${ }^{(15)}$.

Compreende-se que mesmo que o Enfermeiro seja responsável no sentido legal em relação a implementação da SAE nas instituições de saúde, os desafios e dificuldades aqui mencionados, exigem uma reflexão crítica não apenas dos Enfermeiros, mas da equipe de enfermagem, assim como dos funcionários responsáveis por gerenciar as unidades, salientando a importância do compromisso com a assistência de qualidade, a fim de propor medidas e ações que visam minimizar os obstáculos enfrentados para a adesão do método.

\section{Restabelecimento da ordem através da educação continuada}

A correta utilização da SAE como instrumento de auxílio para tomada de decisão, implementação e avaliação da assistência é extremamente relevante para todo o processo de cuidado ${ }^{(16)}$. Por isso, torna-se necessária a capacitação, atualização, supervisão e educação permanente dos profissionais de enfermagem no processo de implantação da SAE nas instituições brasileiras. Além do mais, para as instituições que possuem a SAE implantada, é importante a realização de avaliações periódicas, no intuito de melhorar a assistência, identificar as dificuldades e intervir nos aspectos afetados ${ }^{(17)}$.

Vale ressaltar que os Enfermeiros entendem e reconhecem a Sistematização da assistência como uma ferramenta de valor e eficiente, porém, não a implementam de maneira integral devido as objeções relatadas. De acordo com os resultados coletados, a falta de conhecimento (N.
4; 33,32\%) e a necessidade de aprimoramento (N. 4; 33,32\%) são mencionadas nos periódicos apontando a importância e deficiência na formação e educação permanente da Enfermagem, retardando o processo de implementação, uma vez que o conhecimento teórico age como um mediador no processo de implantação.

Sendo a SAE um dos principais instrumentos para o desenvolvimento da competência clínica do enfermeiro, é fundamental que estes profissionais busquem capacitação no intuito de ampliar seu conhecimento e campo de visão, não se limitando as práticas e demandas cotidianas, bem como o conhecimento adquiridos nos bancos acadêmicos ${ }^{(18)}$.

Há lacunas no que diz respeito ao conhecimento sobre a SAE que os profissionais de enfermagem possuem, sendo necessário aprimorar o ensino desta nas instituições de formação de técnicos de enfermagem e enfermeiros, além de existir a necessidade de estimular a qualificação profissional, a educação permanente, a educação continuada e o treinamento da equipe para utilização dos instrumentos ${ }^{(19)}$.

Este estudo se tornou relevante, pois, retrata os desafios e dificuldades enfrentadas pelos Enfermeiros na implementação da SAE de acordo com a literatura, além das falhas institucionais, estas que quando não corrigidas, podem transparecer de modo negativo sob a forma de uma assistência ineficaz e defasada. Contudo, mesmo diante de tais objeções, é necessário que a equipe de Enfermagem e gerencial se unam para que as açôes educativas sejam adotadas.

\section{CONCLUSÃO}

A aplicabilidade errônea da sistematização da assistência produz um efeito retrógrado no que diz respeito à autonomia que o enfermeiro conseguiu adquirir no seu campo de trabalho caracterizadas pelas dificuldades identificadas desde a formação dos Enfermeiros até condições de trabalho que prejudicam o êxito da execução do método. Destaca-se a necessidade de promoção da educação continuada e aper- 
feiçoamento dos profissionais em relação à aplicabilidade da SAE, no intuito de melhorar de maneira significativa a qualidade assistencial. Em suma, este estudo corrobora aos demais estudos, ao trazer a iden- tificação de algumas perspectivas que contribuem de maneira negativa e retardam a implementação da SAE, incentivando o pensamento crítico a respeito da temáti$\mathrm{ca}$, a fim de propor melhorias em todo o sistema e compreende que os desafios em relação à aplicabilidade da sistematização da assistência são muitos, sendo necessária uma reformulação no âmbito educativo e profissional. -

\section{REFERÊNCIAS}

1. Malucelli A, Otemaler KR, Bonnet M, Cubas MR, Garcia TR. Sistema de informação para apoio à Sistematização da Assistência de Enfermagem Rev Bras Enferm [internet]. 2010 [citado em 10 mar 2021]; 63(4):629-36. DOl: https://doi.org/10.1590/S003471672010000400020

2. Alfaro-LeFevreR. Aplicação do processo de enfermagem: uma ferramenta para o pensamento crítico. $7^{\mathrm{a}}$ ed. Porto Alegre: Artmed; 2010.

3. Conselho Federal de Enfermagem. Resolução 358/2009. Dispõe sobre a Sistematização da Assistência de Enfermagem e a implementação do Processo de Enfermagem em ambientes, públicos ou privados, em que ocorre o cuidado profissional de Enfermagem, e dá outras providências. [Internet]. [citado em 27 Dez 2020]. Disponível em: http:/www.cofen.gov.br/resoluo-cofen-3582009438_4.htm

4. NANDA. Diagnósticos de Enfermagem da NANDA-I. Definições e classificações. 2018-2020. $11^{\text {a }}$ ed. 2018.

5. Linch GFC, Paz AA, Caregnato RCA, Abreu AM, Souza EM. Ações coordenadas para implantação e consolidação da sistematização da assistência de Enfermagem. Enferm. Foco. Brasilia [internet]. 2019 Dez [citado em 27 Dez 2020]; 10(7): 87-93. Disponivel em: https://pesquisa. bvsalud.org/brasil/resource/pt/biblio-1051338

6. Somariva VCA, Birolo IB, Tomasi CD, Soratto J. Percepções das equipes de enfermagem na atenção básica frente à sistematização da assistência de Enfermagem. Enf. Foco. Brasilia [internet]. 2019. [citado em 27 Dez 2020]; 10(4): 142-147. https://pesquisa.bvsalud.org/brasil/ resource/pt/biblio-1052822

7. Marcos ACA, Oliveira JL, Souza J. Percepção da equipe de enfermagem quanto à sistematização da assistência de enfermagem em um serviço de emergência psiquiátrica. REME rev. min. enferm; [internet] 2016. [citado 27 Dez 2020]; 20: [1-6]. Disponível em: https:/pesquisa. bvsalud.org/brasil/resource/pt/biblio-835270

8. Silva EGC, Oliveira VC, Neves GBC, Guimarães TMR. O conhecimento do enfermeiro sobre a Sistematização da Assistência de Enfermagem: da teoria à prática. Rev Esc Enferm [internet]. 2011. Dez [citado em 10 mar 2021]; USP. Dec; 45(6):1380-6. DOI: http://dx.doi.org/10.1590/ S0080-62342011000600015

9. Marinelli NP, Silva ARA, Silva DNO. Revisão de literatura Sistematização da Assistência de Enfermagem - Desafios para a implantação. · Revista Enfermagem Contemporânea. [internet]. 2015 Jul.-Dez [citado em 10 Mar 2021]; 4(2):254-263. DOl: http://dx.doi. org/10.17267/2317-3378rec.v4i2.523

10. Mendes KDS, Silveira RCCP, Galvão CM. Revisão integrativa: método de pesquisa para a incorporação de evidências na saúde e na enfermagem. Texto Contexto Enferm. [Internet]. 2008 [citado em 27 Dez 2020]; 17(4):758-64. Disponivel em: https:/www.scielo.br/scielo. php?script=sci_arttext\&pid=S0104-07072008000400018

11. Medeiros AL, Santos SR, Cabral RWL. Sistematização da assistência de enfermagem: dificuldades evidenciadas pela teoria fundamentada nos dados. Rev. enferm. UERJ [internet]. 2013 jan-mar [citado em 27 Dez 2020]; 21(1): 47-53. Disponivel em: https:/pesquisa.bvsalud.org/ brasil/resource/pt/lil-743013

12. Tavares TS, Castro AS, Figueiredo ARFF, Reis DC. Avaliação da implantação da sistematização da assistência de enfermagem em uma unidade pediátrica. REME rev. min. enferm. [internet] $2013 \mathrm{abr-jun}$ [citado em 27 Dez 2020]; 17(2): 42-50. Disponível em: https:/pesquisa. bvsalud.org/brasil/resource/pt/lil-696398

13. Santos FC, Martins JDN, Souza MWO, Carvalho DNR, Santos APG, Pimentel IMS. Sistematização da assistência de Enfermagem na perspectiva da equipe de enfermagem de um hospital público do norte do Brasil. Nursing (Säo Paulo) [internet] 2019 Set [citado em 27 Dez 2020]; 22(256): 3155-3159. Disponivel em: https:/pesquisa.bvsalud. org/brasil/resource/pt/biblio-1026021

14. Dotto, Jl, Backes DS, Dalcin CB, Lunardi Filho WD, Siqueira $\mathrm{HCH}$, Zamberlan C. Sistematização da assistência de enfermagem: ordem, desordem ou (re)organização?. Rev. enferm. UFPE on line [internet] 2017 Out [citado em 27 Dez 2020]; 11(10): 3821-3829. Disponivel em: https:/pesquisa.bvsalud.org/brasil/resource/pt/biblio-1031884

15. Menezes SRT, Priel MR, Pereira LL. Autonomia e vulnerabilidade do enfermeiro na prática da Sistematização da Assistência de Enfermagem. Rev Esc Enferm USP [internet] 2011 Ago [citado em 27 Dez 2020]; 45(4): 953-8. Disponivel em: https://pesquisa.bvsalud.org/brasil/resource/pt/mdl-21876898

16. Souza VN, Pereira AS, Vesco NL, Brasil BMBL, Barbosa SM, Viana CDMR. Conhecimento das enfermeiras de ambulatórios de hemofilia sobre a sistematização da assistência de enfermagem. Rev. enferm. UFPE on line [internet] 2016 maio [citado 27 Dez 2020]; 10(5): 16541662. Disponivel em: https:/pesquisa.bvsalud.org/brasil/resource/pt/ biblio-1031649

17. Neco KKS, Costa RA, Feijão AR. Sistematização da Assistência de Enfermagem em instituições de saúde no Brasil: revisão integrativa Rev. enferm. UFPE on line [internet] 2015 jan [citado 27 Dez 2020]; 9(1): 193-200. Disponível em: https:/pesquisa.bvsalud.org/brasil/resource/pt/biblio-998793

18. Machado JPC, Silva DM, Souza E, Pedron CD, Gallasch CH, Thiengo PCS. Percepção de enfermeiros de unidades de internação clínica sobre a Sistematização da Assistência de Enfermagem Nursing (Säo Paulo) [internet] 2019 Out [citado 27 Dez 2020]; 22(257): 3220-3225. Disponivel em: https:/pesquisa.bvsalud.org/brasil/resource/pt/biblio-1026095

19. Mola Rm Dias ML, Costa JF, Fernandes FECV, Lira GG. Conhecimento dos profissionais de enfermagem sobre a sistematização da assistência de enfermagem. Rev. pesqui. cuid. Fundam [internet] 2019 Jul-Set [citado 27 Dez 2020]; 11(4): 887-893. Disponivel em: https:// pesquisa.bvsalud.org/brasil/resource/pt/biblio-1005652 